\title{
Feasibility, Limitations and Potentiality of UHF-RFID Passive Implants
}

\author{
C. Occhiuzzi, M. Simiele, R. Lodato, G. Marrocco \\ Pervasive Electromagnetics Lab, University of Roma Tor Vergata, \\ Via del Politecnico, 1 \\ 00133, Roma (ITALY) \\ Email: occhiuzzi@disp.uniroma2.it marrocco@disp.uniroma2.it
}

\begin{abstract}
Implanted RFID may play an important role in the personal Healthcare of next future. Antenna embedded into prosthesis or into other implanted medical devices could permit to monitor physiological and pathological processes, providing a natural interconnection to remote services. The big technical challenge is to establish a stable RFID link with the interior of the human body in spite of the high electromagnetic losses of the tissues. By using parametric electromagnetic models and some early result, it is here investigated the potentiality and limitation of the UHF implants with special attention to the body district, the overall mass, and the size of the antennas.
\end{abstract}

\section{INTRODUCTION}

Health is one of the priorities that the OECD (Organization for Economic Co-operation and Development) countries are asked to face in the next future. The demographic development, the progressive increase of the percentage of elderly people, the growth of chronic degenerative diseases following the current lifestyles (smoking, obesity, and inactivity) contribute toward the need of innovative healthcare solutions, based on decentralized, pervasive and patient-centric approaches. Engineering and Medical technologies should share expertise and efforts to create a body-centric network of knowledge and care where persons, their internal organs and even their implanted medical devices are continuously and remotely monitored in order to have access every time and everywhere to information about their condition and state. A strategic role in the present and future healthcare could be played by wearable and implanted medical devices such as prosthesis or drugs delivery systems. Their importance in restoring physiological functions and, definitely, in saving lives, actually prompt the need of a continuous monitoring of their really effectiveness so providing the clinicians with useful information to evaluate the success of their action and accordingly to update the medical treatment, or more drastically to prevent severe drawbacks for the patients in case of complications and failure. Some solutions have been proposed just in the very last years but they are generally subjected to the presence of complex electronics components: one or more active sensors, power supply, transceiver and antenna. The resulting devices are not completely bio-compatible, may suffer from local failure and, not least, they provide limited lifetime or need for power recharge.

An alternative and promising technology is now emerging from new progresses of Radio Frequency Identification (RFID)
[1]. Together with its ID code widely used for logistics, an RFID tag is in fact able to carry information about the tagged object such as its physical state and its evolution along with time, without any specific embedded sensor or local power supply [2]. This kind of "transparent", non-intrusive devices could offer great advantages in medicine: one or more batteryless RFID IC could be integrated inside clothes [3], [4] or into implanted medical devices provided with conductive elements (prosthesis, sutures, stents or orthopedic fixings) such to transform them into RFID radio-sensors suitable to be interrogated by an external reader and able to "sense" themselves and the close surrounding environment. By means of data acquisition at different times, it will be possible to collect the history of geometrical or chemical features of tissues and devices, thus observing in an indirect way the functioning, the healing process and possible complications [5].

One of the first attempts to implant RFID passive device in the human body for only identification purposes was due to Very-Chip tag [6]. More elaborate experiments, also suitable to sensing applications, generally involve active or semiactive devices usually equipped with chemical or mechanical sensors [7], super capacitors or other components for energy harvesting. In general, RFID implants also suffer of poor link range [8] due the high loss affecting the electromagnetic interaction with the human body.

Although these problems have been extensively investigated in the LF and HF band (inductively coupling operating in $\mathrm{HF}$ is now one of the most common methods to wirelessly send power and data from off-body interrogator to RF implants), the Ultra-High Frequency (UHF) band is pretty unexplored, leaving in doubt its effective benefits or limitations. Several theoretical studies investigated the dependency of the antenna's parameters on the locus of the implants or on the human variability [8], [9]. Such efforts experimentally demonstrated [10] that it is possible to read a passive tag implanted into a neck-like phantom up to $20 \mathrm{~cm}$ with $0.5 \mathrm{~W}$ of transmitted power. However many aspects remain in question.

Within this scenario, the aim of the work is to provide a parametric study of the communication link between an RFID tag implanted in the human body and the external reader. By means of numerical simulations and early invitro experiments, some useful normalized indicators will be introduced with the aim to understand the effective feasibility 
of passive RFID implants (achievable read ranges, minimum antenna's size, required powers and sensitivities), the actual technological limitations and the potential, providing firstlevel guidelines for the design of future passive Body centric Implanted Network.

\section{Numerical Model}

In the UHF band the human body, essentially composed of water, bones and tissues, can be viewed as a highly scattering and dissipative electromagnetic propagation channel in which multiple reflections (multi-paths) and strong attenuation occur. In order to perform an analysis of the UHF wireless communication, a realistic electromagnetic model of the human body (with its various tissues and geometries) has to be taken into account. Homogeneous or simple-shaped phantoms are the simplest way to simulate the human body and to produce qualitative information on the implanted antennas. At the same time, progresses in medical imaging technologies, e.g. MRI, $\mathrm{X}$-Ray and CT, have encouraged instead the development of precision-head and whole- body voxel models [11]. Such models offers greater accuracy but require larger computational cost and are not easily reproducible in-vitro. Usually, contrary to simple-shape models, voxel-models refer to standard body configurations, e.g male/female, adult/child, thin/stout subject, they are not scalable and not able to represent particular pathologies, such as obesity, cancer, the presence of other medical implants or the functionality of the different organs, e.g. inflated/deflated lugs, empty/filled stomach or bladder. At this purpose a dynamic Finite Difference Time Domain (FDTD) model has been proposed, able to provide good accuracy but at the same time to take benefit of the simplicity, the scalability and the in vitro reproducibility of simple-shaped phantoms.

Starting from anatomical human models and medical images, the numerical body phantom has been set up by means of basic shapes (cylinders, spheres) able to well-approximate the most relevant external and internal structures (Fig.1). The model is focused on the human torso that is one of the most complex region from the anatomical and electromagnetic perspective and one of the most concerned by implanted devices [12]. Each organ, whose shape and size resemble the real ones, is characterized by its dielectric properties [13] in the UHF band and is able to reproduce physiological or pathological conditions by changing of size and material. Also the overall dimensions of the phantoms can be scaled such to easily represent different body form factors as well as global conditions such as the obesity.

\section{RFID EQUATIONS AND LINK's PARAMETERS}

The RFID communication exploits a two-way link: a direct one, wherein the key-issue is the scavenging of power at the chip's port so that the tag may activate and perform actions, and a reverse link wherein the tag transmits its data to the reader by changing its reflectivity through impedance modulation. The link [14] for UHF-RFID systems (overall World-wide band: $866-956 \mathrm{MHz}$ ) is commonly characterized

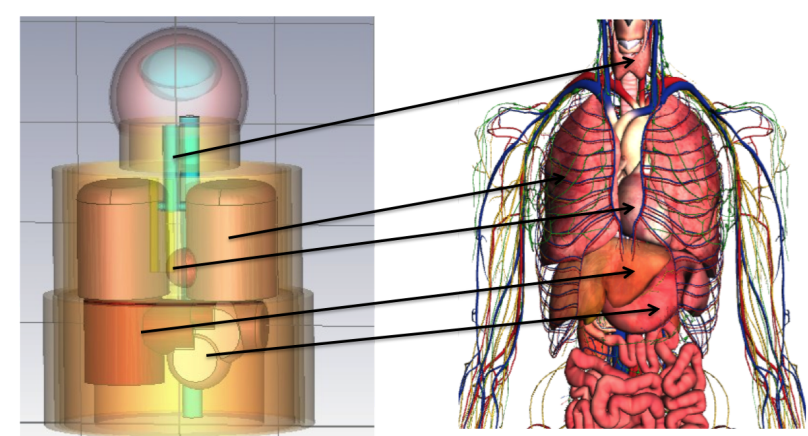

Figure 1. FDTD and Anatomical Human Model.

under the far field assumption by using the Friis formula for the direct path (1), and the radar cross-section for the reverse one (2):

$$
\begin{gathered}
P_{R \rightarrow T}=\left(\frac{\lambda_{0}}{4 \pi d}\right)^{2} P_{i n} G_{R}(\theta, \phi) G_{T}(\theta, \phi) \tau \eta_{p} \\
P_{R \leftarrow T}=\frac{1}{4 \pi}\left(\frac{\lambda_{0}}{4 \pi d^{2}}\right)^{2} P_{i n} G_{R}^{2}(\theta, \phi) \eta_{p}^{2} r c s_{T}(\theta, \phi)
\end{gathered}
$$

$d$ is the reader-tag distance, $G_{R}(\theta, \phi)$ is the gain of the reader antenna, $G_{T}[\theta, \phi]$ is the gain of the tag's antenna. $P_{i n}$ is the power entering the reader's antenna, $\eta_{p}$ is the polarization mismatch between the reader and the tag, and $\tau$ is the power transmission coefficient of the tag :

$$
\tau=\frac{4 R_{\text {chip }} R_{a}}{\left|Z_{\text {chip }}+Z_{a}\right|}
$$

with $Z_{\text {chip }}$ input impedance of the RFID integrated circuit (IC) and $Z_{a}$ input impedance of the antenna. $r c s_{T}$ is the tag's radar cross-section, related to the modulation impedance $Z_{\text {mod }}$ of the microchip to encode the low and high digital states:

$$
r c s_{T}=\frac{\lambda_{0}^{2}}{4 \pi} G_{T}^{2}(\theta, \phi)\left(\frac{2 R_{a}}{\left|Z_{\text {mod }}+Z_{a}\right|}\right)^{2}
$$

Generally, the bottleneck of the whole tag-reader communication is imposed by the direct link, due to the rough chip power sensitivity (currently of the order of $10^{-5} \mathrm{~W}$ ) and by the allowed emitted power from the reader by local regulations, which hence limits the maximum read range. The parameter that describes the tag's scavenging capabilities is instead the realized gain $G_{\tau}=G_{T} \tau$, e.g the gain of the tag's antenna scaled by the mismatch to the RFID IC. It directly imposes the maximum read ranges and thus the possibility to interrogate from outside an implanted RFID tag. The maximum read distance $d_{\max }$ is computed from (1) by enforcing $P_{R \rightarrow T}=P_{c h i p}$, i.e the IC's sensitivity:

$$
d_{\text {max }}(\theta, \phi)=\frac{\lambda_{0}}{4 \pi} \sqrt{\frac{P_{i n} G_{R}(\theta, \phi) G_{T}(\theta, \phi) \tau \eta_{p}}{P_{\text {chip }}}}
$$


For the whole reader implanted-tag system, the allowed maximum read range will be investigated in the next sections with respect to the antenna position and size, the IC sensitivities, the power transmitted by the reader and the human body variability.

\section{Parametric Analysis}

The design of an antenna suitable for implanted RFID sensor tags is a challenging issue in itself since it should be compliant with both electromagnetic and anatomical requirements and eventually to the prosthesis wherein the tag is attached or integrated. For the sake of generality, a reference dipole antenna has been considered for the study. As demonstrated in [10], the field produced by an half-wavelength dipole implanted in the human body can be approximated by the "far-field" assumption just after a distance of about $15 \mathrm{~cm}$ from the locus of the implant, and hence it is possible to apply the standard RFID equations to extract the communication parameters. Since such a distance is comparable also with the sizes of other body districts, such as the abdominal region, the same considerations can be extended to the whole human body.

\section{A. Position}

The input impedance and radiation pattern of the implanted tag may depend on the location of the antenna inside the body [15]. Five different locations of the gastrointestinal tract have been therefore considered (Fig.2). The gastrointestinal apparatus, from the pharynx to the stomach, is one of the most complex and in-homogeneous from the electromagnetic perspective, since it reaches different depths $s$ in the body (rather superficial in the neck while deeper in the abdominal region) going through tissues with extremely different dielectric properties (lung-liver). Furthermore, it is the locus of the implant of many devices already adopted or scientifically envisaged, such as endoscopic capsules, surgical drains and bariatric prosthesis for the gastric bypass.

The reference tag is a flat dipole provided with an inductor to balance the imaginary part of the IC input impedance; the reader is assumed to emit $3.2 \mathrm{~W}$ EIRP and the chip sensitivity is fixed to $P_{\text {chip }}=-18 \mathrm{dBm}$.

A first simulation set considers a $L=2 \mathrm{~cm}$ dipole. A strong decrease of the FDTD-computed resulting realized gain is visible in Fig.3, moving from the most superficial position (neck $s_{1}=7 \mathrm{~cm}$ ) to the deepest one (abdomen $s_{4}=20 \mathrm{~cm}$ ). The increased size of the phantom and the deeper position of the radio-sensor are indeed responsible of a rise of the losses and consequently of the power required to establish the communication. In the worst case of implant inside the abdomen, the power sensitivity of off the shelf RFID IC $\left(P_{\text {chip }}=-18 \mathrm{dBm}\right)$ doesn't allow a robust communication link between the external reader and an implanted passive RFID device.

Two conditions have been evaluated for the stomach: the empty and filled states [8]. When the organ is empty, the tag is surrounded by air and thus an increase of the radiation

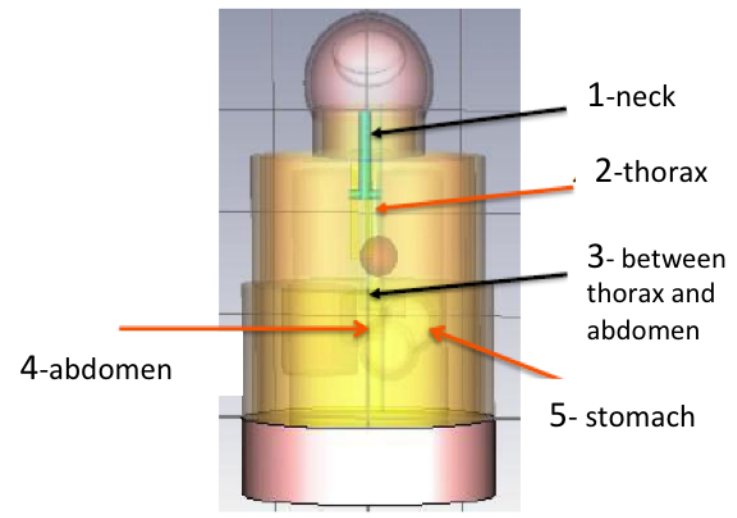

Figure 2. Five different body districts for tag implantation. Depths: $s_{1}=$ $7 \mathrm{~cm}, s_{2}=13 \mathrm{~cm}, s_{3}=18 \mathrm{~cm}, s_{4}=20 \mathrm{~cm}, s_{5}=18 \mathrm{~cm}$.

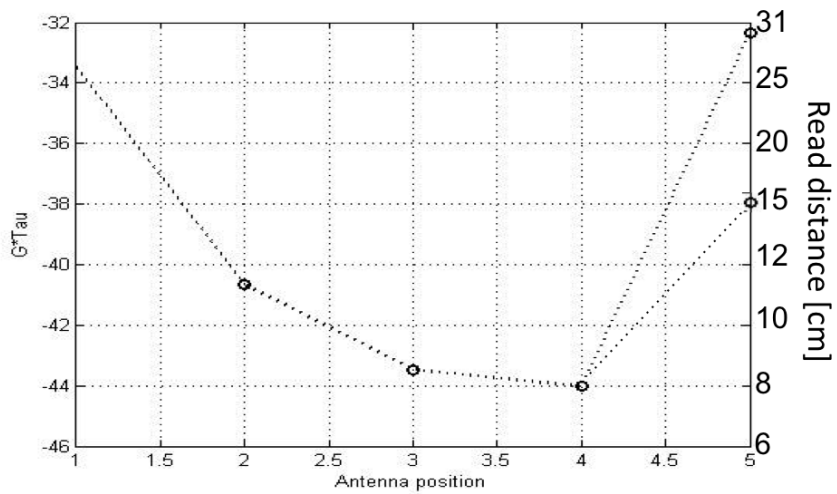

Figure 3. Realized gain $G_{\tau}$ and maximum achievable read ranges (in $\mathrm{cm}$ ) for a $L=2 \mathrm{~cm}$ dipole tag, by varying the locus of implantation. For position 5 (stomach) two different operative conditions have been evaluated, i.e. stomach full and empty. Here 3.2W EIRP and $P_{\text {chip }}=-18 d B m$.

performance similar to the ones of the neck is registered in spite of the position is much more deep. As soon as the stomach fills up, a $6 \mathrm{~dB}$ attenuation occurs, since the losses of the stomach's content (a mixture of food and gastric juice) become predominant.

\section{B. Antenna Size}

Like conventional antennas, also for the RFID tag the radiation performances depend on the antenna's size. Although one of the most pressing requirements is the small size of the radiating element, larger radiators may be integrated with prothesis, sutures, orthopedic fixing and vascular stents, eventually taking advantage from their conducting structures. Starting from $L=2 \mathrm{~cm}$, the length of the flat-dipole implanted in the neck has been progressively increased up to almost twice its initial size. As expected, the read range increases as the dipole becomes longer (Fig.4), with an average rate of approximately $2 \mathrm{~dB} / \mathrm{cm}$.

It is interesting to notice that the improvement to the read distance given by the size of the radiator is even more negligible in case the tag is implanted deep in the body, e.g. in the stomach (position 5 in Fig.2). As shown in Fig.5, an increase 


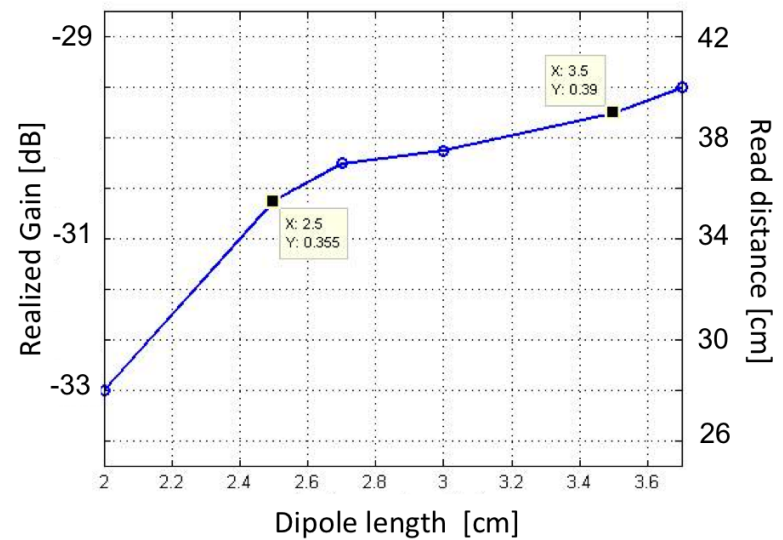

Figure 4. Realized gain $G_{\tau}$ and maximum achievable read distance (in $\mathrm{cm}$ ) for the flat dipole implanted in position 1 (neck) by varying its length. In this case EIRP $=3.2 \mathrm{~W}$ and $P_{\text {chip }}=-18 \mathrm{dBm}$.

of $4 \mathrm{~cm}$ in the dipole length produces an enlargement of almost $9 \mathrm{~cm}$ in the read distance, with an average rate of approximately $0.55 \mathrm{~dB} / \mathrm{cm}$. For the largest antenna's configuration, the trend becomes even not linear. Such results would confirm that for deep implants, i.e. bariatric prosthesis, the communication link is governed more by the path loss, e.g. the attenuation due to the propagation in the human tissues, than by the antenna's features.

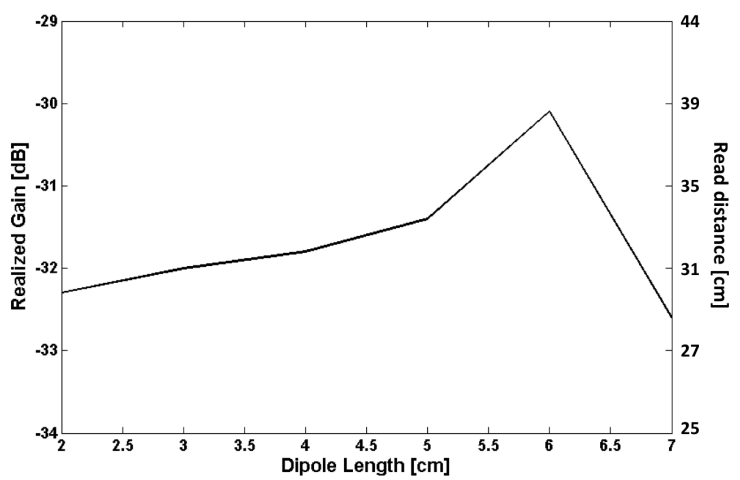

Figure 5. Realized gain $G_{\tau}$ and maximum achievable read distance (in $\mathrm{cm}$ ) for the flat dipole implanted in position 5 (stomach) by varying its length. In this case EIRP $=3.2 \mathrm{~W}$ and $P_{\text {chip }}=-18 \mathrm{dBm}$.

\section{IC Sensitivity}

The maximum allowed read distance is dependent, from (5), on the IC power sensitivity with an approximate trend of $3 \mathrm{~cm} / \mathrm{dB}$ (Fig.6). It it worth recalling that due to the advances in low-power electronics, a reduction of about two orders of magnitude of the IC sensitivity has been achieved in the last 10 years [16] and thus it is reasonable to expect very soon to correctly interrogate the smallest $2 \mathrm{~cm}$ tag in the stomach which requires an IC sensitivity less than $P_{\text {chip }}=-21 \mathrm{dBm}$.

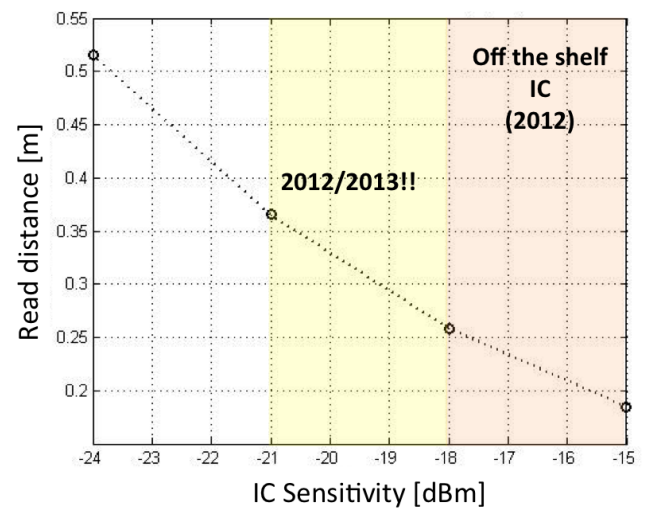

Figure 6. Maximum read distance for the flat dipole by varying the IC sensitivity. In this case $\mathrm{EIRP}=3.2 \mathrm{~W}, \mathrm{~L}=2 \mathrm{~cm}$, Position 1 (neck)

\section{Body conformation}

The RFID link is also dependent on the body conformation, and in particular on the pathological presence of fat in the abdominal region, such in case of an obese patient. At this purpose, the radiation performances of a $2 \mathrm{~cm}$-long dipole implanted in position 4 of Fig. 3 have been studied by progressively increasing the thickness of the abdominal fat and hence the overall size of the numerical phantom. Although fat is one of the human tissue with lowest permittivity and conductivity due to its low water content, a gain reduction of almost $12 \mathrm{~dB}$ is observed (Fig.7), moving from an healthy subject (fat thickness $2 \mathrm{~cm}$ ) up to an obese subject (fat thickness 10 $\mathrm{cm})$. The trend is almost linear, with a rate of approximately $-1.5 \mathrm{~dB} / \mathrm{cm}$

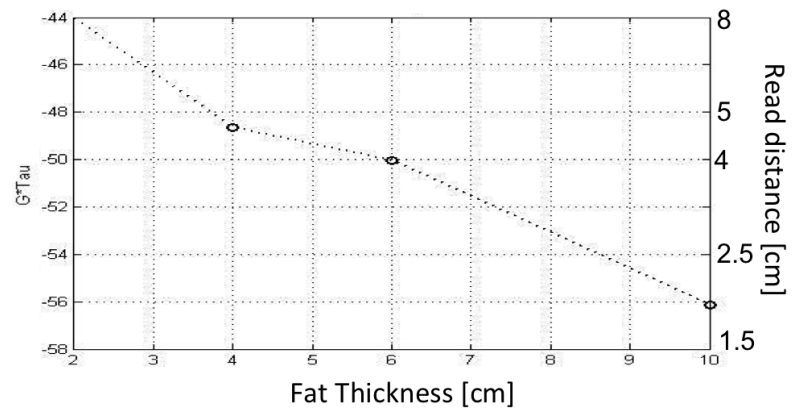

Figure 7. Realized gain $G_{\tau}$ and maximum achievable read distance (in $\mathrm{cm}$ ) by varying the fat thickness of the abdominal region. In this case $\mathrm{L}=2 \mathrm{~cm}$. Position 4 (abdomen).

For such class of patients the communication is hence very challenging. To overcome such problems, IC with extremely low sensitivity are required, together with antennas with larger sizes such to achieve a greater gain.

\section{EARLY EXPERIMENTATION}

A preliminary experimental characterization has been performed by means of an equivalent liquid phantom emulating the first section of the gastrointestinal apparatus, e.g the neck region (Fig.8). The neck is represented by a $2 \mathrm{~cm}$ thick perspex 
cylinder, with diameter $D=13 \mathrm{~cm}$ and height $h=20 \mathrm{~cm}$ filled by a homogeneous dielectric having the weighted average permittivity of all the main tissues composing the neck $\left(\bar{\epsilon}_{\text {neck }}=41.4-j 14.8\right)$, while a smaller off-centered $3 \mathrm{~cm}$ diameter cylinder simulates the human esophagus and it is filled by air [10]. The liquid phantoms have been obtained with mixtures of water, sugar and salt according to the recipes in [17]. The sizes of the phantom have been chosen such to reproduce the overall characteristics of the numerical model in Section II. In particular the height of the cylinder comprises also the bulk of the head and part of the shoulders. All the measurements have been performed within the UHF band (840-960 MHz) by means of Thing-Magic M5e reader connected to a $5 \mathrm{~dB}$ gain linear polarized patch antenna (Fig. 8 ), which is placed at $20 \mathrm{~cm}$ from the neck phantom.

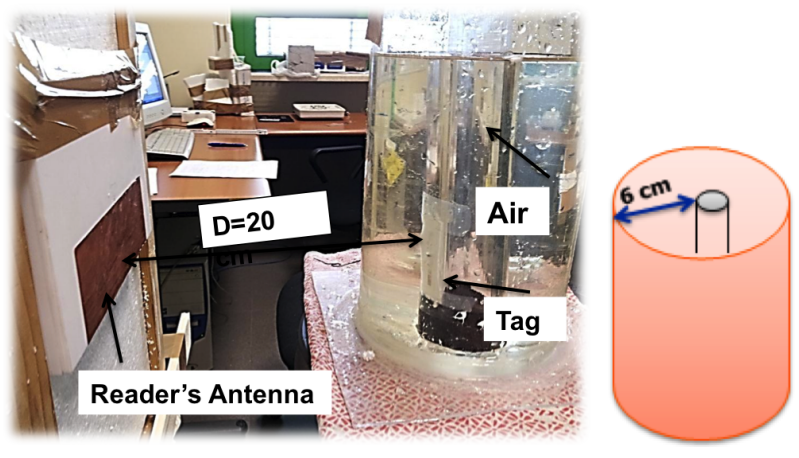

Figure 8. Equivalent Liquid phantom and its schematic representation

The tag prototype is a $3 \mathrm{~cm}$ dipole (Fig. ) integrating a seriesinductor to match the IC NXP-G2XL $\left(Z_{\text {chip }}(870 \mathrm{MHz})=\right.$ $\left.15-j 135 \Omega, P_{\text {chip }}=-15 \mathrm{dBm}\right)$. The tag has been insulated from the phantom liquid by glued PCV sheets.

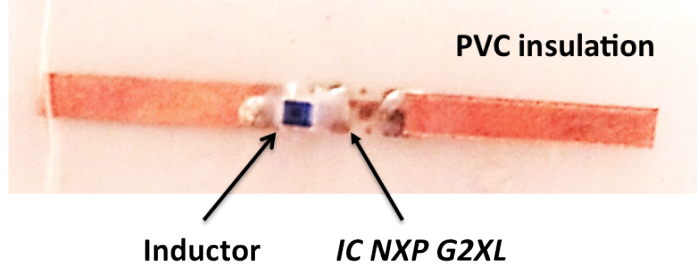

Figure $9 . \quad 3 \mathrm{~cm}$ implanted tag

The effect of the different implantation districts has been roughly reproduced by changing the position of the esophaguslike pipe with respect to the phantom border. For example, two implant depths have been considered $s_{A}=3 \mathrm{~cm}$ (a rather superficial implant) and $s_{B}=6 \mathrm{~cm}$ (resembling the locus $s_{1}$ in Fig.2). The corresponding realized gain, as estimated from the turn-on measurements, is shown in Fig.10. The different depth of the implants induces a $2 \mathrm{~dB}$ difference in the realized gain throughout the considered $850-960 \mathrm{MHz}$ band. In particular, the measurement for $s_{B}=6 \mathrm{~cm}\left(G_{\tau}\left(s_{B}\right)=\right.$ $-27 d B @ 870 \mathrm{MHz})$ is fully comparable with the simulated result (Fig.4) referred to a slightly different implant distance $s_{1}=7 \mathrm{~cm}\left(G_{\tau}\left(s_{1)}=-30 \mathrm{~dB}\right)\right.$.

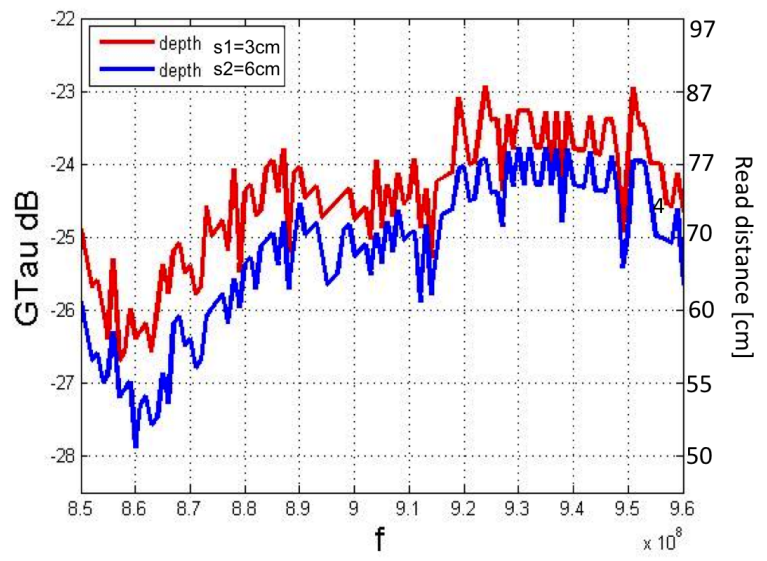

Figure 10. Realized gain and estimated maximum read distance (3.2 W EIRP and $P_{\text {chip }}=-18 \mathrm{dBm}$ ) by varying the locus of implantation.

\section{CONCLUSION}

Numerical investigations and early experiments have highlighted the conditions to establish an RFID link between an external reader and an implanted tag in several districts of the gastrointestinal apparatus. The link is affected by human and electromagnetic parameters, mostly the locus of the implant and the IC sensitivities. In some conditions, the nowadays technology seems to limit the possibility to use UHF passive tags, within the allowed power emission, for deep implant such as in the abdominal area. However, good improvements are expected by considering the rapid advantages in low power electronics.

Finally, it is worth noticing that the analysis so far presented are related only on the tag's side of the system. For a full understanding of the limitations and potentials, also the performance of the reader in close proximity of the human body needs investigation as well. Some advances will be presented at the Symposium.

\section{ACKNOWLEDGMENT}

The authors wish to thank prof. Nicola Di Lorenzo for inspiration and useful discussions.

\section{REFERENCES}

[1] D. M. Dobkin, The RF in RFID: passive UHF RFID in Practice. Amsterdam: Elsevier, 2007

[2] G. Marrocco, "Pervasive electromagnetics: sensing paradigms by passive rfid technology," Wireless Communications, IEEE, vol. 17, no. 6, pp. 10 -17 , december 2010.

[3] C. Occhiuzzi and G. Marrocco, "The rfid technology for neurosciences: Feasibility of limbs' monitoring in sleep diseases," Information Technology in Biomedicine, IEEE Transactions on, vol. 14, no. 1, pp. 37 -43, jan. 2010.

[4] C. Occhiuzzi, S. Cippitelli, and G. Marrocco, "Modeling, design and experimentation of wearable rfid sensor tag," Antennas and Propagation, IEEE Transactions on, vol. 58, no. 8, pp. 2490 -2498, aug. 2010.

[5] C. Occhiuzzi and G. Marrocco, "Sensing the human body by implanted rid tags," in Antennas and Propagation (EuCAP), 2010 Proceedings of the Fourth European Conference on, april 2010, pp. 1 -5.

[6] K. Foster and J. Jaeger, "Rfid inside," Spectrum, IEEE, vol. 44, no. 3, pp. $24-29$, march 2007. 
[7] E. Chow, Y. Ouyang, B. Beier, W. Chappell, and P. Irazoqui, "Evaluation of cardiovascular stents as antennas for implantable wireless applications," Microwave Theory and Techniques, IEEE Transactions on, vol. 57, no. 10, pp. $2523-2532$, oct. 2009.

[8] A. Sani, M. Rajab, R. Foster, and Y. Hao, "Antennas and propagation of implanted rfids for pervasive healthcare applications," Proceedings of the IEEE, vol. 98, no. 9, pp. 1648 -1655, sept. 2010.

[9] L. S. Xu, M. Q.-H. Meng, and C. Hu, "Effects of dielectric values of human body on specific absorption rate following 430, 800, and $1200 \mathrm{mhz}$ rf exposure to ingestible wireless device," Trans. Info. Tech. Biomed., vol. 14, no. 1, pp. 52-59, Jan. 2010. [Online]. Available: http://dx.doi.org/10.1109/TITB.2009.2029853

[10] C. Occhiuzzi, G. Contri, and G. Marrocco, "Design of implanted rfid tags for passive sensing of human body: the stentag," Antennas and Propagation, IEEE Transactions on, vol. PP, no. 99, p. 1, 2012.

[11] P. S. Hall and Y. Hao, Eds., Antennas And Propagation for Body-Centric Wireless Communications, 1st ed. Norwood: Artech House Publishers, August 2006.

[12] H. Rajagopalan and Y. Rahmat-Samii, "Ingestible rfid bio-capsule tag design for medical monitoring," in Antennas and Propagation Society International Symposium (APSURSI), 2010 IEEE, july 2010, pp. 1 -4.

[13] http://www.brooks.af.mil/ AFRL/HED/hedr/reports/dielectric/home.html, C.Gabriel, S.Gabriel, Compilation of the Dielectric Properties of Body Tissues at RF and Microwave Frequencies, http://www.brooks.af.mil/ AFRL/HED/hedr/reports/dielectric/home.html.

[14] P. Nikitin and K. Rao, "Theory and measurement of backscattering from rfid tags," Antennas and Propagation Magazine, IEEE, vol. 48, no. 6 , pp. $212-218$, dec. 2006.

[15] J. Kim and Y. Rahmat-Samii, "Planar inverted-f antennas on implantable medical devices: Meandered type versus spiral type," Microwave and Optical Technology Letters, vol. 48, no. 3, pp. 567-572, 2006. [Online]. Available: http://dx.doi.org/10.1002/mop.21409

[16] G. Marrocco, "Rfid antennas for the uhf remote monitoring of human subjects," Antennas and Propagation, IEEE Transactions on, vol. 55, no. 6 , pp. $1862-1870$, june 2007.

[17] G. Hartsgrove, A. Kraszewski, and A. Surowiec, "Simulated biological materials for electromagnetic radiation absorption studies." Bioelectromagnetics, vol. 8, no. 1, pp. 29-36, 1987. [Online]. Available: http://www.ncbi.nlm.nih.gov/pubmed/3579997 